characteristic phasing is just what would be expected if the horizontal sections had exposed the $\mathrm{M}$ and $\mathrm{E}$ oscillators. This new approach, and its attendant change in perspective, holds the promise of understanding the basis of seasonal rhythms.

But have Jagoda et al. really identified the $\mathrm{M}$ and $\mathrm{E}$ oscillators? Several arguments support this possibility. First, when slices were taken from hamsters exposed to longer days than nights (a light: dark cycle of 14:10 versus 8:16), the onset of the morning peak was advanced and its duration lengthened relative to the evening peak; the phases of the two peaks are not mutually locked. Second, the two peaks respond in the expected way to a pulse of glutamate, the neurotransmitter that normally conveys light signals from the eye to the SCN. Thus, glutamate given after dusk delayed the evening peak but not the morning peak, whereas glutamate given before dawn advanced the morning but not the evening peak. Nevertheless, although these findings are consistent with independent behavioral output from dual oscillators, they are not sufficient to prove the case-and indeed the authors are careful to avoid committing themselves as to whether their two peaks actually represent the $M$ and E oscillators. For proof, one would like to see distinct outputs from the two oscillators, to confirm that they can display different period lengths, and to see them run across each other assuming in passing all possible phase angles. Ideally, one would also like to be able to manipulate the two peaks individually through genetic or pharmacological means and confirm that they really are independent. To do all this in an in vitro preparation whose viability is limited will be challenging, to say the least.

There are excellent precedents in the animal literature for dual separable oscillators. Neuronally coupled clocks have been described in the optic lobes of the cockroach (Leucophaea maderae) ${ }^{8}$, and in the eyes of marine molluscs such as Aplysia or Bulla (for example, see refs 9 and 10), and data—both physiological and pharmacological — consistent with dual oscillators in the SCN have been around for years (for example, refs. 1 and 5). If dual oscillators are present in the SCN but haven't been seen, then something either lost or gained in the horizontal section is exposing them. Both are possibilities, because distinct subregions exist within the SCN that have distinct neuropeptide and gene expression profiles (for example, see ref. 11). Moreover, connections within the SCN are complex, so the consequences of cutting the tissue at different angles are hard to predict. For instance, the first clock - specific impact of light in the SCN appears to be the induction of Per1 in an area of the ventrolateral SCN that receives inputs from the reti$\mathrm{na}^{12}$. From here, the effect of light on the clock gradually moves to the dorsolateral $\mathrm{SCN}$, whose cells do not receive a direct input from the retina. It is plausible that neuronal connections between the paired SCN lie dorsally, in the part that is removed in the horizontal plane of sectioning but that is retained in the coronal section (Fig. 1). Then again, the typical coronal section removes aspects of the caudal and rostral extent of the SCN, where yet another oscillator could lie, and the connections that might tie the most rostral parts of the paired SCN are not fully understood. Three-dimensional reconstructions have suggested that hamster SCNs are actually fused posteriorally ${ }^{13}$. Even if they aren't actually joined in other species, that they are in hamsters suggests that there are likely to be tight connections in all species, and that these connections could be lost in coronal sections.

Unravelling all these connections within the circuitry of the SCN should be great fun for all concerned, but what of the broader significance? I believe that Jagota et al. ${ }^{7}$ is a major step forward, in that it succeeds in bridging the behaviorally-based model of the $\mathrm{E}$ and $\mathrm{M}$ oscillators with cellular/tissue level phenomena that are likely to point to its anatomical and physiological underpinnings. It doesn't prove the existence of the E and M oscilla- tors in the isolated SCN, but it does raise interesting and important questions, at least some of which are now made more experimentally tractable. The conceptual leap in this work could drive a branch of circadian experiments over much of the next decade, and one can only agree with the authors' understated conclusion: "We predict that the horizontal slice preparation will prove to be a useful experimental system". Yup, it probably will.

1. Klein, D. C., Moore, R. Y. \& Reppert, S. M. Suprachiasmatic Nucleus: The Mind's Clock (Oxford, New York, 1991).

2. Welsh, D. K., Logothetis, D. E., Meister, M. \& Reppert, S. M. Neuron 14, 697-706 (1995).

3. Herzog, E., Takahashi, J. \& Block, G. Nat. Neurosci. 1, 708-713 (1998).

4. Earnest, D. J., Liang, F. Q., Ratcliff, M. \& Cassone, V. M. Science 283, 693-695 (1999).

5. Gillette, M. U. et al. Ciba Found. Symp. 183, 134-144 (1995).

6. Pittendrigh, C. S. \& Daan, S. J. Comp.Physiol. A 106, 333-355 (1976).

7. Jagota, A., de la Iglesia, H. O. \& Schwartz, W. J. Nat. Neurosci. 3, 372-376 (2000).

8. Page, T. Science 216, 73-75 (1982).

9. Block, G. D., Geusz, M., Khalsa, S. \& Michel, S. Sem. Neurosci. 7, 37-42 (1995).

10. Eskin, A. Fed. Proc. 38, 2573-2579 (1979).

11. Moore, R. Y. Prog. Brain Res. 111, 103-119 (1996).

12. Shigeyoshi, Y. et al. Cell 91, 1043-1053 (1997).

13. Lydic, T., Albers, H. E., Teppers, B. \& MooreEde, M. C. J. Comp. Neurol. 20, 225-237 (1982).

\title{
Which way, honey?
}

Evidence indicates that men perform better than women at spatial navigation tasks, such as finding their way in an unfamiliar environment. On page 404, Riepe and colleagues now establish a neural correlate for this gender difference, by showing that men and women show different patterns of brain activation during navigation. The authors asked subjects to navigate their way out of a virtual reality maze displayed on a computer

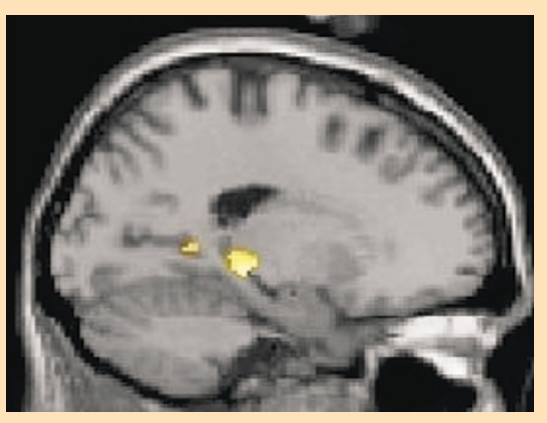
while their brain activity was monitored by fMRI. As anticipated from previous work, they found that men are faster than women at finding their way out of the maze. However, although some brain areas were equally activated in men and women, there were several differences. Men showed activation of the left hippocampal region (which was previously shown to be involved in spatial tasks) whereas women showed activation of the parietal and right prefrontal cortices. The significance of this differential activation is less clear, but the results raise the possibility that men and women may be using different cognitive strategies to solve the navigation task. Now, could this have anything to do with the notorious male reluctance to ask for directions?

Kalyani Narasimhan 